\title{
High Power Density and High Efficiency Converter Topologies for Electric Ships
}

\author{
J.D. Herbst, F.D. Engelkemeir, and A.L. Gattozzi \\ Center for Electromechanics \\ The University of Texas at Austin \\ Austin TX USA \\ j.herbst@cem.utexas.edu
}

\begin{abstract}
Power electronic converters are expected to be significant contributors to system mass, system loss, and system cost in the all-electric ship, and are therefore a significant area of interest. This paper investigates the reduction of switching losses in high power (MW level) converters. These losses remain a major obstacle to the development of converters capable of operating at higher frequencies and higher power densities.
\end{abstract}

The Auxiliary Resonant Commutated Pole (ARCP) softswitching converter topology offers the potential for minimization of switching losses but has some inherent limitations. This paper examines two new converter designs based on the ARCP soft-switching topology that allow for more compact units by reducing the semiconductor switching losses generated within them. These concepts have been proven in principle by preliminary laboratory testing of a scaled $20 \mathrm{~kW}$ converter prototype. The new proposed topologies are described and simulation results and experimental waveforms obtained on the prototype unit are also reported.

Keywords-Converter topology, ARCP, Auxiliary Resonant commutated Pole, Switching losses

\section{INTRODUCTION}

Advanced electric sensors and weapons are rapidly increasing the demand for electric power on naval platforms. As part of the Electric Ship Research and Development Consortium (ESRDC), The University of Texas Center for Electromechanics (UT-CEM) has been conducting research into integrated electric power systems with support from the Office of Naval Research (ONR) and the Naval Surface Warfare Center, Carderock Division (NSWCCD). Much of the research has centered on the system-level evaluations of power system architectures and identification of high payoff technology development activities. In these advanced power system concepts, ship hotel, weapon, and propulsion loads will require power with different voltage and frequency characteristics and power electronic conversion equipment will be a critical element of the power distribution system. ESRDC architecture evaluations show that power conversion equipment can represent $25-30 \%$ of the total volume and weight of the electric power distribution system [1] and the number of conversion stages has a significant impact on overall efficiency of the system. Enhanced power conversion efficiency has broader implications on ship design as the reduced losses can potentially reduce the size of the associated thermal management system. Increasing the capability, power density, and efficiency of power conversion equipment will have a major impact on the space allocation aboard a ship and on the performance of the power system. In support of this goal, UT-CEM is actively developing new power converter concepts that will improve the performance and efficiency of these critical elements of naval electric power systems. This paper reports on two novel topologies for improved power conversion.

\section{BASELINE TOPOLOGY}

UT-CEM is exploring the application of resonant, softswitching converters which aim at minimizing losses by turning the semiconductor devices on or off at zero voltage or zero current: this should result, in theory, in zero switching loss transitions for the devices. Switching losses in the semiconductors are a large fraction of the total converter losses and, in fact, become the dominant loss component at the higher operating frequencies envisioned for advanced ship power system concepts [2]. The Auxiliary Resonant Commutated Pole (ARCP) topology [3] is one of the most promising ones from this standpoint and UT-CEM has developed and demonstrated a 2 MW ARCP prototype converter [4] that is now an element of the Microgrid Laboratory at UT-CEM.

The novelty of the ARCP converter lies entirely in its inverter section. Its schematic diagram is shown in Figure 1. The ARCP inverter is composed of a standard six leg inverter section (main switches S1and S2) which is aided in its switching transitions by an auxiliary section, with auxiliary 
switches A1 and A2 and resonating inductors Lr. It is fundamental to the operation of an ARCP inverter to turn the main switches $\mathrm{S} 1$ and $\mathrm{S} 2$ on when the voltage across them is zero and to turn the auxiliary switches A1 and A2 off when the current through them is zero. The symmetric transitions, turning $\mathrm{S} 1$ and $\mathrm{S} 2$ off or turning $\mathrm{A} 1$ and $\mathrm{A} 2$ on, are already expected to take place at zero voltage or zero current because of their timing in the cycle and because they are further aided by the reactive elements in the circuit, respectively the resonant capacitors and the in-line inductors. This results in theoretically lossless switching in all cases. It should also be noted that a further benefit of the ARCP converter and indeed all soft-switching converters is their potential to reduce the effects of Electromagnetic Interference (EMI) as documented in [5].

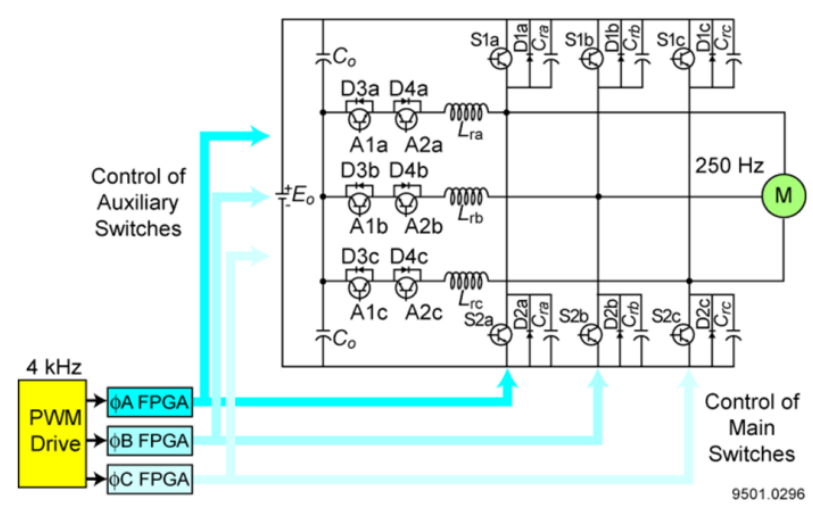

Figure 1. Schematic diagram of the inverter section of the ARCP converter of UT-CEM

The ARCP commutation sequence has been described in the literature [3] and is summarized here for convenience:

a. Starting with main switch $\mathrm{S} 1$ of a given phase conducting $(\mathrm{ON})$ and all other switches non-conducting (OFF); let us examine the controller's commutation from $\mathrm{S} 1$ to $\mathrm{S} 2$ (S1 turns OFF and S2 turns $\mathrm{ON}$ ).

b. Auxiliary switch A2 of the same phase is turned ON (at zero current) and half of the DC DC-bus voltage is applied across Lr: this causes the auxiliary current through Lr to ramp up linearly. The amount of time that Lr is charging is referred to as the "boost time".

c. At the end of the boost time, main switch S1 is turned OFF. The capacitor across S1 holds the voltage at S1 at zero volts temporarily which means $\mathrm{S} 1$ is switched off at zero voltage.

d. The current flowing through Lr acts like a current source that, along with the resonant capacitors, forces the output voltage (at the point of connection to the motor load) to swing from the positive to the opposite negative supply rail. For this to take place in all conditions, the auxiliary current peak must always exceed the load current flowing through the motor phase. e. Once the output voltage hits the negative supply rail, the antiparallel diode in S2 starts to conduct, at which point the gate control logic detects the zero voltage across S2 and turns ON S2 (at zero-voltage).

f. Now both A2 and S2 are turned ON and a voltage of half the supply voltage is again applied across Lr, but now of the opposite polarity. This causes the current in Lr to decrease linearly.

g. Once the current in $\mathrm{Lr}$ has decreased to zero, the auxiliary switch A2 is turned OFF at zero current.

Figure 2 reports simulation and actual experimental waveforms obtained for the UT-CEM $20 \mathrm{~kW}$ converter prototype built according to the standard ARCP topology.
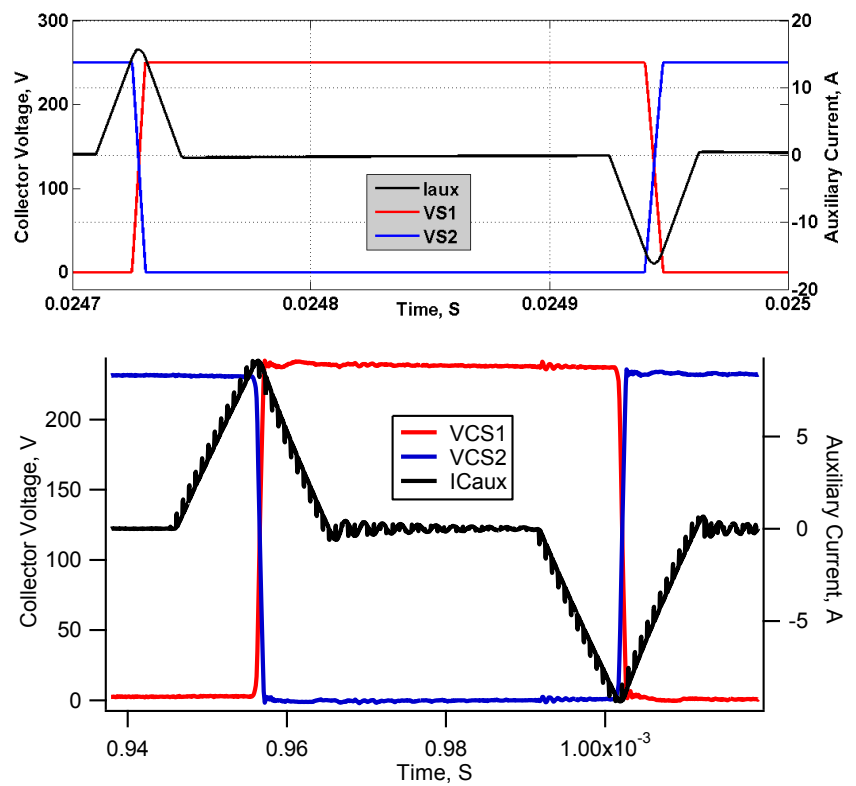

Figure 2. Simulation results (upper traces) and experimental waveforms (lower traces) for the standard ARCP converter. (For both: main switch S1 is red, main switch S2 is blue, and the auxiliary current is black).

Note that a potential equal to half of the dc bus voltage is required for the auxiliary circuit. This can either be provided by a center-tapped dc bus (as in the converter at UT-CEM in Figure 1) or a small capacitive voltage divider may be provided exclusively for the use of the auxiliary circuit [6]. In either case, the voltage balance of the two capacitors must be maintained with additional circuitry or with an intelligent gating control.

Also note that the auxiliary current always flows through a diode (the anti-parallel/freewheeling diode of the nonconducting complementary auxiliary IGBT) as well as the IGBT which is actually turned $\mathrm{ON}$ in the auxiliary branch. These diodes exhibit a reverse-recovery current that is somewhat "snappy", leading to a large di/dt. This, in turn, causes the inductor that carries the same current to generate a voltage spike across the auxiliary switches that must be taken into account to avoid switch failure [7]. Each of the auxiliary 
IGBTs switches handles the entire auxiliary current for the respective switching cycle, and its antiparallel diode is also carrying the entire auxiliary current for the time that the complementary auxiliary switch is conducting.

The standard ARCP topology has been investigated at length at UT-CEM and its shortcomings have been reported [8]. Analytical and experimental work on the larger $2 \mathrm{MW}$ ARCP prototype has led to the development of two new concepts for modified ARCP power converter topologies that eliminate the performance limitations identified with the original configuration.

\section{NEW ARCP CONCEPT \#1}

The first proposed alternative is to modify the ARCP topology by deriving the auxiliary current excitation directly from the dc bus rails as shown in Figure 3. The new topology essentially consists of a main inverter driven by an auxiliary inverter into soft switching transitions at commutation. In this design, the auxiliary inverter is itself soft-switched for half of its transitions.

Powering the auxiliary switches directly from the dc bus effectively doubles the voltage driving the auxiliary current, resulting in larger pulses and, therefore, easier commutations and larger load handling capacity. Alternatively, the boost time can be cut in half while still achieving the same auxiliary current.

Additionally, there is the practical advantage of not having to split the dc bus to generate a neutral point. This neutral point must be monitored and actively balanced for proper operation of the converter, which will require additional control circuitry.

Another major advantage of the new architecture is the fact that the auxiliary current now does not flow through a diode and, therefore, no diode reverse recovery current must be accounted for and no additional snubbers are necessary.

Also, now both ends of the inductor are tied to the center point of half-bridges that are built with power devices containing freewheeling diodes, and as such, the voltages are fully clamped to be within the dc bus voltage rails.

In this topology, each auxiliary switch conducts the entire auxiliary current for its respective switching cycle during auxiliary current ramp-up. For the second half of the cycle the opposite auxiliary switch's anti-parallel diode conducts the auxiliary current during the Lr ramp-down discharge. Thus the conduction losses of this topology are less than those of the standard ARCP since the auxiliary switches are only conducting for half the time, relative to the standard ARCP where both of the auxiliary switch devices are conducting (either the auxiliary IGBT, or the anti-parallel diode of its complementary device) for both the auxiliary current ramp up and ramp down time.

A prototype of this topology has been tested showing that it is realizable with only one remaining drawback, namely that one transition per cycle of the auxiliary switches has to remain hard-switched, as will be explained below.

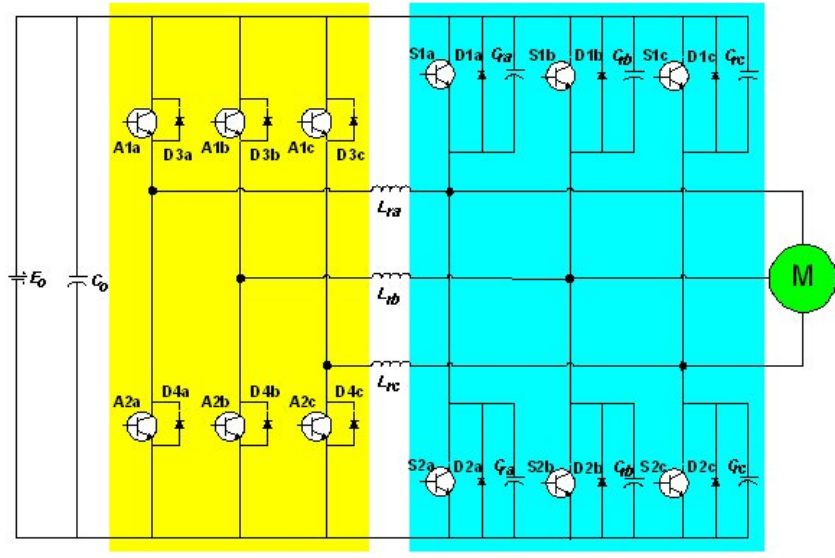

Figure 3. Proposed new topology \#1 for ARCP converter.

The detailed commutation sequence for this topology will now be described following the same sequence used for the standard ARCP.

a. Start with main switch S1 ON and all other switches OFF and with the controller starting commutation from S1 to S2.

b. Auxiliary switch A2 is turned ON (at zero current) and the full dc bus voltage is applied across $\mathrm{Lr}$ to cause the auxiliary current to ramp up linearly.

c. At the end of the boost time, main switch S1 is turned OFF at zero voltage thanks to the capacitor across S1.

d. The current flowing through Lr acts like a current source that, along with the resonant capacitors, forces the output voltage to swing to the opposite supply rail.

e. Once the voltage hits the negative supply rail, the antiparallel diode in S2 starts to conduct, the gate control logic detects the zero voltage across S2 which is turned $\mathrm{ON}$ at zero-voltage.

f. f. Now both A2 and S2 are turned ON and there is a current flowing in a loop through $\mathrm{Lr}$ that must be interrupted. This is done by turning A2 OFF with a hardswitched event and the energy stored in Lr is returned to the de bus capacitors via A1's anti-parallel diode.

As mentioned previously, this sequence includes the hard-switched turn-off of an auxiliary IGBT (last step listed above). This hard-switching event cannot be avoided, as the current carried by the IGBT has no opposing voltage in its path and flows through the inductor $\mathrm{Lr}$ and a very low resistance loop: therefore, its decay time constant is typically much longer than the converter switching cycle.

Figure 4 reports both simulated and experimental waveforms obtained on this topology. The main switches S1 and S2 voltage traces show that the switching action of the main switches are the same as for the standard ARCP shown in Figure 2. 

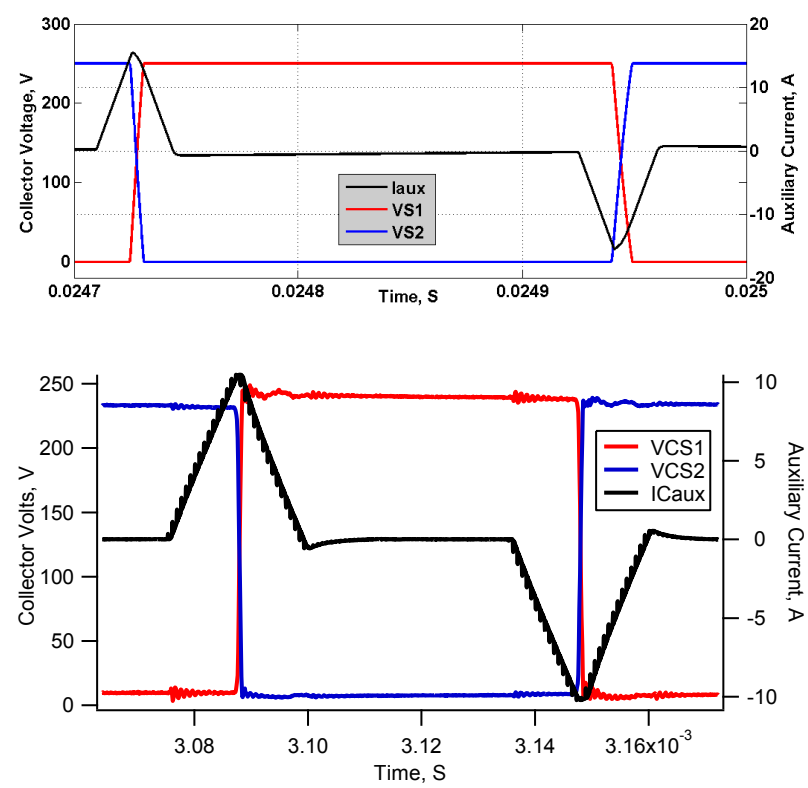

Figure 4. Simulated and measured waveforms for the new concept no. 1 ARCP topology (same color code as in Figure 2).

\section{NEW ARCP CONCEPT \#2}

To address this remaining limitation, UT-CEM designed and tested a second alternative topology shown in Figure 5. Here a virtual neutral is again generated by the addition of a transformer stage in the auxiliary circuit. The virtual neutral point emulates the split-capacitor of the standard ARCP. This virtual neutral point is again necessary to connect the auxiliary circuit to a mid-point voltage such that when the main switches S1 and S2 commutate, the voltage across the resonant inductor can be reversed allowing its discharge.

With this configuration it has been proven that all transitions can be achieved in a soft-switching mode without the drawbacks of the standard ARCP topology. When one of the auxiliary switches is activated, current flowing in one winding of the transformer (from the center tap that is connected to Lr) induces an equal flow in the other transformer winding, causing the anti-parallel diode of the complementary auxiliary switch to conduct. This in effect puts the entire bus voltage across the transformer, with the center tap reflecting a voltage level equal to half the supply voltage. In this mode of operation the auxiliary switches only see half of the auxiliary current, relative to the standard ARCP where the auxiliary switches are directly in series with the auxiliary circuit and see the entire current, allowing them to be made smaller. Each auxiliary device will have half of the auxiliary current flowing through them (either the switch proper or its anti-parallel diode) due to the transformer action. The transformer's magnetic core does need to be reset after each switching cycle. This can be achieved with a diode across the primary of the transformer (not shown in the diagram of Figure 5 for simplicity).

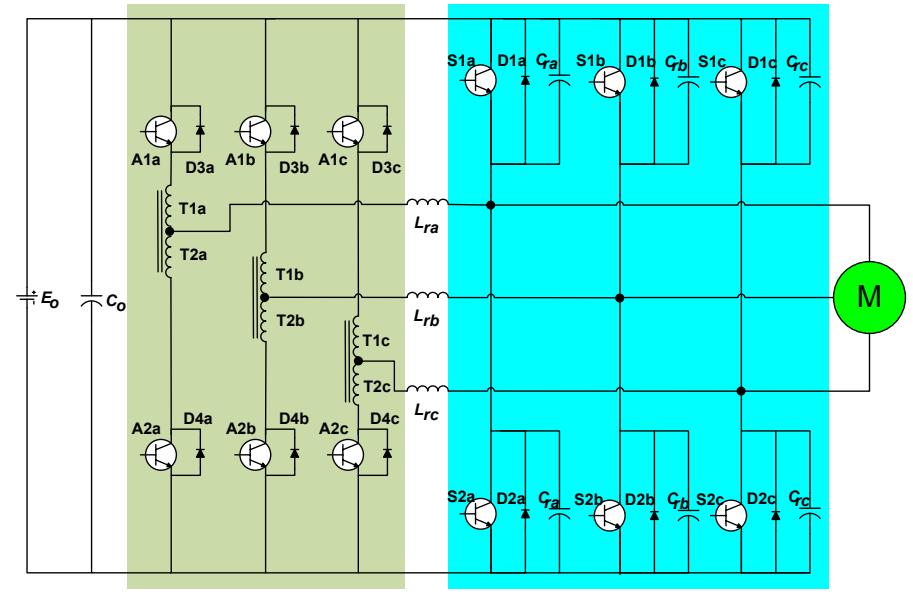

Figure 5. New ARCP Concept \#2

Following the same pattern used above, the ARCP Concept \#2 commutation sequence is described below.

a. $\quad \mathrm{S} 1$ is $\mathrm{ON}$ and the control initiates commutation to $\mathrm{S} 2$

b. A2 is turned $\mathrm{ON}$ and current begins to flow through $\mathrm{Lr}$ and into T2. Due to the series inductance limiting current flow during turn-on, this is a zero-current turn-on. Because of transformer action, an equal current begins to flow through A1's anti-parallel diode, which clamps the top point of the transformer to the positive supply rail. The transformer has the full bus voltage across it, and due to the 1:1 winding ratio, the center-tap is at half of the supply voltage. Thus Lr has one half of the supply voltage applied across it causing the boost current to ramp-up. The currents in $\mathrm{A} 1$ and $\mathrm{A} 2$ are equal and are one half of the current in Lr. The inverter is in this state for an amount of time equal to the boost time.

c. At the end of the boost time period, when a large enough current has built up in Lr, S1 is turned OFF. The $\mathrm{Cr}$ capacitor maintains the voltage at zero across $\mathrm{S} 1$ during the S1 switch-off to create a zero-voltage turn-off. The current through $\mathrm{Lr}$ is now flowing out of the $\mathrm{Cr}$ capacitor and causes the output voltage to swing to the opposite supply rail. The duration of this swing is a function of the boost current, load current, and the value of $\mathrm{Cr}$.

d. The output voltage eventually slews down to the negative supply rail and forward biases the anti-parallel diode in S2. S2's zero-voltage detector will detect this condition and turn $\mathrm{S} 2 \mathrm{ON}$.

e. S2 is turned ON under zero-voltage conditions. The inverter has successfully transitioned to the S2 ON state, but the auxiliary inductor must now be discharged. When the output switched from S1 to S2, the voltage on the right hand side of Lr switched from the positive supply rail to the negative supply rail. The voltage at the center tap of the transformer is still half the supply voltage so that Lr has half of the supply voltage connected across it but in the opposite polarity, causing a ramp down of the current. This is very similar to the standard ARCP. The 
current will continue to ramp down for a period of time approximately equal to the boost time.

f. Once the current in Lr has hit zero Volts, A1 is switched OFF with zero voltage (A2's anti-parallel diode also stops conducting). This completes the commutation sequence. Commutation from S2 back to S1 is the same except that the complementary auxiliary switch is used.

Simulated and experimental waveforms are reported in Figure 6. The main switches S1 and S2 voltage traces show that the switching action of the main switches are the same as for the standard ARCP. The Auxiliary current traces show that each auxiliary device A1 and A2 carry half of the auxiliary current.
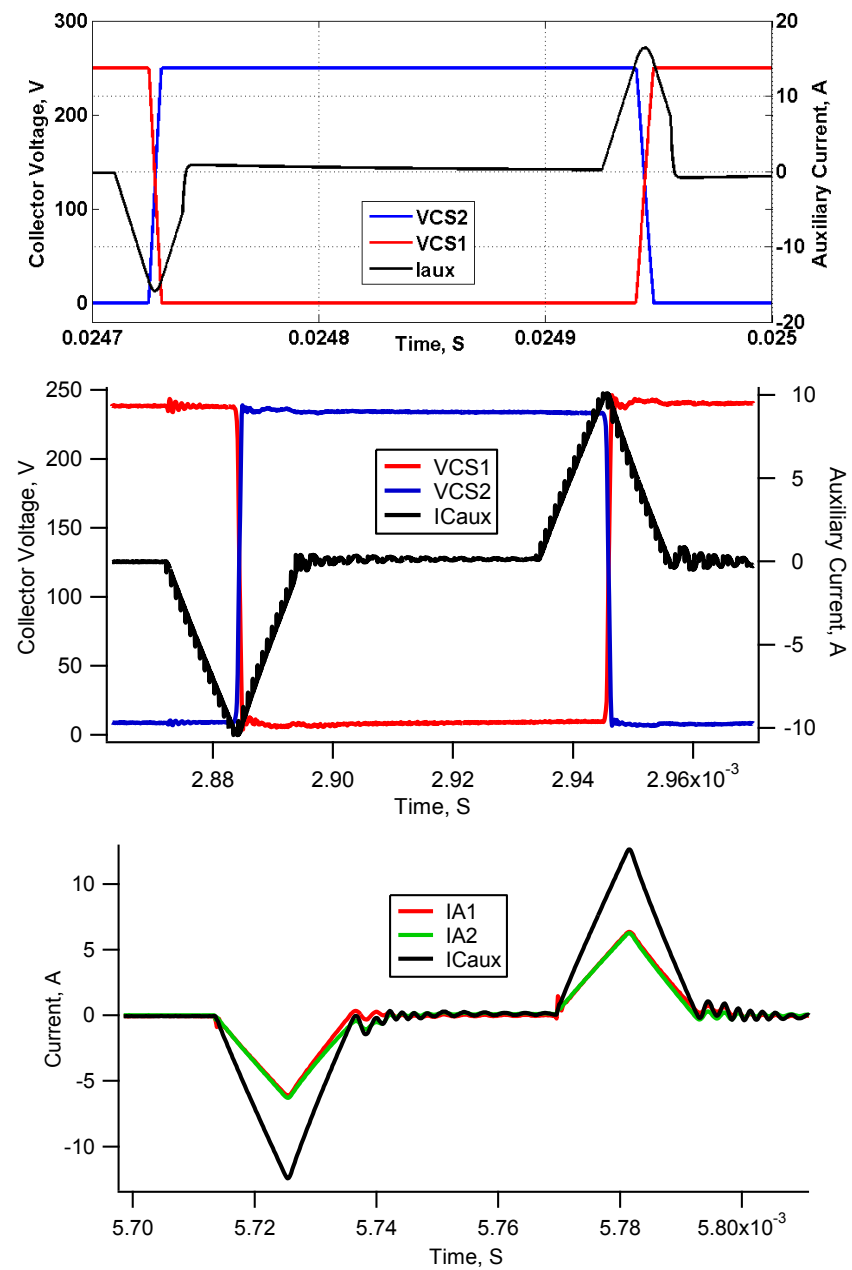

Figure 6. Simulated and measured waveforms for the new concept no. 2 ARCP. The final plot shows the current through auxiliary switches A1(red) and A2(green) summing to the auxiliary current (black).

\section{SimUlations AND EXPERIMENTAL PROTOTYPE}

MATLAB/Simulink with the SimPowerSystems package was used for the simulations.

A three phase $20 \mathrm{~kW}$ experimental inverter that can be configured in any of the three discussed topologies was constructed using PowerEX CM100DY-24NF IGBT modules driven by PowerEX VLA504 gate driver modules. Zero- current and zero-voltage detection was provided by discrete circuitry. Xilinx' Complex Programmable Logic Devices (CPLDs) were used to generate the soft-switching gating signals. A Baldor S15 drive was used to generate the PWM waveforms. The switching frequency was set to $4 \mathrm{kHz}$ to match the operating conditions of the 2 MW ARCP converter. $130 \mu \mathrm{H}$ air core inductors were used for the standard ARCP and the new ARCP concept \#2. $260 \mu \mathrm{H}$ air core inductors were used for the ARCP new concept \#1 to keep the timing and operating conditions identical. A $10 \mu \mathrm{s}$ boost time was used, and the resonant capacitors were $10 \mathrm{nF}$ film-type capacitors rated for snubber duty. The experimental prototype is shown in Figure 7.

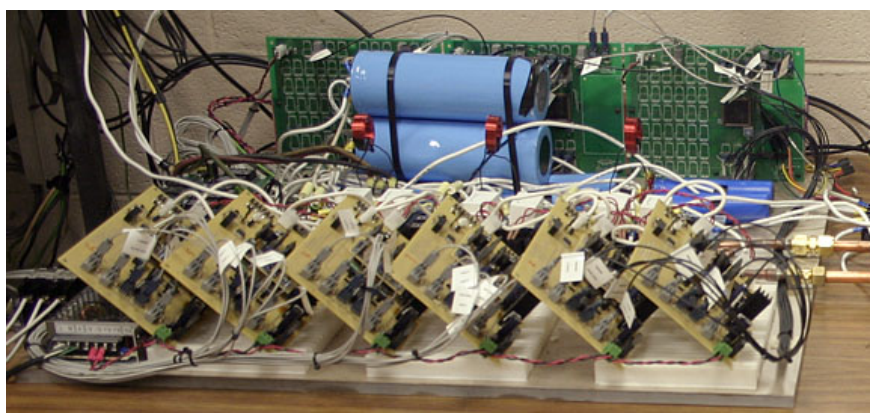

Figure 7. $20 \mathrm{~kW}$ experimental inverter

\section{DISCUSSION AND RESULTS}

In summary, the proposed new topologies for a soft switching converter achieve the following primary goals:

- By using the full supply voltage for commutation, the first alternative topology maximizes the achievable power density with better efficiency than the conventional ARCP, with soft switching in all transitions except one remaining hard-switched transition in the auxiliary switches.

- The second alternative topology maximizes the converter efficiency achieving full soft-switching although with a lower power density than the first alternative topology since once again only half the dc bus is available for commutation. Furthermore, a small pulse transformer is needed.

A full comparison of the three topologies is captured in Table I.

The differences in the three topologies are in the auxiliary circuit: the main switches and their soft-switching commutation is identical. New concept \#1 can be used to increase the power handling of the ARCP inverter or reduce its size but with residual switching losses in the auxiliary devices. New concept \#2 allows the ARCP to be operated without the need for a split bus and the associated extra capacitors and balance control issues, and allows potential further size reductions in the auxiliary switches due to each device handling half of the auxiliary current, but adds the need for a small pulse transformer and the need to reset its magnetized core. 
TABLE I. SUMMARY COMPARISON OF THE THREE TOPOLOGIES

\begin{tabular}{|c|c|c|c|}
\hline & Standard ARCP & New concept \#1 & New Concept \#2 \\
\hline Main devices softswitched & yes & yes & yes \\
\hline Auxiliary devices soft-switched & yes & partially & yes \\
\hline Split DC bus required & yes & no & higher \\
\hline Power handling & normal & no & yes \\
\hline Transformer required & no & no & Transformer reset \\
\hline Voltage spike issue & On auxiliary switches & half \\
\hline $\begin{array}{c}\text { Auxiliary device current(total device, IGBT and } \\
\text { freewheel diode) }\end{array}$ & full & $\begin{array}{c}\text { Full (for half the } \\
\text { time) }\end{array}$ & \\
\hline
\end{tabular}

It must be observed that in all these designs the softswitching is induced by the auxiliary circuit. This means that, in the event of a fault, the converter can continue to operate in the hard-switched mode (albeit at reduced efficiency or power output) in the main circuitry, provided the control circuitry is properly programmed to handle this event.

Finally, it is to be noted that the improvements described above with the new topologies are applicable to either siliconbased converters or to converters using other semiconductor families, like $\mathrm{SiC}$ or $\mathrm{GaN}$ semiconductors. In fact, these latter families of devices, when coupled with the soft-switching ARCP operation, will afford even better performance than achievable with hard-switched technology.

A comparison of the efficiencies of the three converter designs was attempted on the $20 \mathrm{~kW}$ prototype. However, the losses in these inverters are very small (approximately around $2 \%$ of rated power), so that a small measurement error in the power reading translates into a large error in the converter loss and, as a consequence, its calculated efficiency.

Although a high performance Voltech 6000 wattmeter was used in the measurements, the results so far have been inconclusive. Additional refinements of the measurement technique are planned in order to obtain definitive efficiency values.

\section{CONCLUSIONS}

The work done to date at UT-CEM for the development of a viable ARCP converter has been reported in this paper. The standard topology has been compared with two new topologies.

The proposed new configurations for a soft switching ARCP converter have been proven to be viable alternatives and achieve the following goals:

- The first alternative topology maximizes the achievable power density with better efficiency than the conventional ARCP albeit with one remaining hardswitched transition in the auxiliary circuit.

- The second alternative topology maximizes the converter efficiency achieving full soft-switching with the same power density as the standard topology and none of the limitations.
Simulation results have been reported and compared with experimental data obtained on a $20 \mathrm{~kW}$ prototype. The plan is to transition the new topologies onto the larger $2 \mathrm{MW}$ ARCP converter in order to be able to validate their proper functioning at relevant power levels and quantify their anticipated gains in efficiency.

\section{REFERENCES}

[1] "ONR Shipboard Electrical Architecture Study Request for Information", Report of ESRDC to ONR, Dec. 11,

[2] Guennegues, V.; Gollentz, B.; Meibody-Tabar, F.; Rael, S.; Leclere, L.; , "A converter topology for high speed motor drive applications," Power Electronics and Applications, 2009. EPE '09. 13th European Conference on, vol., no., pp.1-8, 8-10 Sept. 2009

[3] De Doncker, R.W.; Lyons, J.P.; , "The auxiliary resonant commutated pole converter," Industry Applications Society Annual Meeting, 1990., Conference Record of the 1990 IEEE , vol., no., pp.1228-1235 vol.2, 7-12 Oct. 1990

[4] Williams, A.; Gattozzi, A.; Thelen, R. F.; , "Applications of an Auxiliary Resonant Commutated Pole Converter," Applied Power Electronics Conference, APEC 2007' Twenty Second Annual IEEE, vol., no., pp.661-665, Feb. 25 2007-March 12007

[5] Dong, W.; Choi, J.-Y.; Li, Y.; Boroyevich, D.; Lee, F.C.; Lai, J.; Hiti, S.; , "Comparative experimental evaluation of soft-switching inverter techniques for electric vehicle drive applications," Industry Applications Conference, 2001. Thirty-Sixth IAS Annual Meeting. Conference Record of the 2001 IEEE, vol.3, no., pp.1469-1476 vol.3, Sept. 30 2001-Oct. 42001

[6] Beukes, H.J.; Enslin, J.H.R.; Spee, R.; "Active snubber for high power IGBT modules," AFRICON, 1996., IEEE AFRICON 4th, vol.1, no., pp.456-461 vol.1, 24-27 Sep 1996

[7] Takano, H.; Domoto, T.; Takahashi, J.; Nakaoka, M.; "Auxiliary resonant commutated soft-switching inverter with bidirectional active switches and voltage clamping diodes," Industry Applications Conference, 2001. ThirtySixth IAS Annual Meeting. Conference Record of the 2001 IEEE vol.3, no., pp.1441-1446 vol.3, Sept. 30 2001-Oct. 42001

[8] Herbst, J.D.; Thelen, R.F.; Gattozzi, A.L.; Williams, A.S.; "Experimental results and design guidelines derived from the testing of a $2 \mathrm{MW}, 250 \mathrm{~Hz}$, auxiliary resonant commutated pole bi-directional converter," Applied Power Electronics Conference and Exposition, 2008. APEC 2008. Twenty-Third Annual IEEE, vol., no., pp.1240-1246, 24-28 Feb. 2008 